\title{
STRUCTURAL STUDIES OF CARBON NANOTUBES AND RELATED MATERIALS BY NEUTRON AND X-RAY DIFFRACTION
}

\section{J. DORE}

School of Physical Sciences, University of Kent, Canterbury, UK

\section{A. BURIAN}

Institute of Physics, University of Silesia, Katowice, Poland

AND S. TOMITA

Kobe University, Kobe, Japan

X-ray and neutron diffraction measurements are presented for a number of carbon samples prepared by different techniques. The information obtained from diffraction studies is presented in relation to other types of investigation, particularly in relation to electron microscopy. The characteristics of the graphene sheets are discussed in relation to these features.

PACS numbers: 61.10.-i, 61.12.-q, 61.46.+w

\section{Introduction}

The standard allotropes of elemental carbon have been known for many centuries and the tetrahedral structure of diamond, based on $s p^{3}$ bonding, and the planar hexagonal structure of graphite, based on $s p^{2}$ bonding, occurs in most elementary textbooks on inorganic chemistry. It is only over the last two decades that a new interest in the structural characterisation of alternative forms of carbon has developed with the discovery of fullerene and nanotube structures. Various fabrication methods have now been developed to produce these materials in sufficient quantities for detailed investigation. A number of fascinating results have emerged from these studies, particularly for carbon nanotubes and it has been widely predicted that their use in various applications will have as dramatic an impact on commercial innovation as that resulting from the discovery of semiconductors.

Most of the existing data on the structural properties of carbon nanotubes is based on the use of electron microscopy, which is capable of producing high-resolution images of the nanotubes. These results have shown clearly the formation 
of successive layers of graphitic sheets, which are believed to be in the form of concentric cylinders, although some workers have suggested alternative connecting features. The arrangement of the carbon atoms in the curved layers takes the form of a hexagonal structure, which is not simply related to the cylindrical axis and can adopt a range of helical structures. Although high resolution transmission electron microscopy (HRTEM) techniques can reveal important information on the primary features, it is desirable to obtain qualitative information on the spatial correlations for the whole sample and this can best be achieved by complementary methods based on diffraction studies. The present paper reports on current developments using new techniques centred on international facilities. The work is in progress and the following sections represent a brief survey of current activities, illustrated by a few recent examples.

\section{Fabrication techniques for carbon nanotubes and related materials}

Carbon nanotubes were first produced by Iijima in 1991 and extracted from the deposits produced in a carbon arc discharge tube. Various groups have since used this method to obtain similar material but it is believed that the final product, after solvent extraction and purification, may depend critically on the conditions of formation. Since that time, alternative methods have been developed to produce nanotube material, based on chemical vapour deposition (CVD), using a cobalt or nickel catalyst as an initiator. Electron microscopy has revealed a wide range of morphologies depending on the methods of formation. In this context, it becomes important to use diffraction methods to obtain a characterisation of the atomic arrangement of carbon atoms in these materials. The fabrication methods used by the different groups are listed below:

a) Oxford nanotubes

A conventional method using an arc discharge was used to produce a suffciently large quantity of material for neutron studies (approximately $350 \mathrm{mg}$ ). The sample was purified by standard oxidation procedures and a brief report of the measurements has already been published [1]; further studies using X-ray diffraction are currently being analysed.

b) Namur nanotubes

Several samples have been provided by Lazarescu et al. [2] using CVD methods with a cobalt catalyst, which remains in the sample material. Both neutron and X-ray measurements have been made and will provide an important indication about whether the catalyst contribution to the diffraction pattern can be subtracted to give data on the nanotubes alone. Work is proceeding on the treatment of these datasets.

c) Japanese nanotubes

A novel CVD method has been used by Kyotani [3] to produce carbon nanotubes in a mesoporous alumina membrane. Both neutron and X-ray measurements have been made on the nanotubes in the membrane but the data analysis is proving difficult due to a phase transition in the alumina arising from the high temperature in the deposition process. A preliminary analysis of the data shows that there is partial orientation of the nanotubes in the mesopores. Orientation effects are also 
expected in the spongy samples of carbon nanotubes produced in an arc discharge arrangement by Ando et al. [4] but diffraction data have not yet been obtained for these interesting samples.

d) Carbon onions

An unusual form of carbon may be produced by the heat treatment of diamond nanoparticles [5]. Satoshi and collaborators have shown that the transformation process goes through several stages and one of the intermediate phases corresponds to the formation of material which appears to have the form of a set of spherical graphitic layers. Preliminary X-ray results for this material are given in Sec. 4.3. These examples show that carbon nanotubes may have many different forms depending on their formation. It is the purpose of the diffraction investigations to try to isolate the way in which these differences manifest themselves at the atomic level.

\section{Structural characterisation}

\subsection{Diffraction formalism}

A diffraction pattern is formed from the coherent interference of waves scattered from all atoms in the sample material. In the case of crystalline solids with a periodic lattice, this gives a series of sharp Bragg peaks corresponding to the Fourier components of the spatial distribution. In the case of disordered materials such as glasses and liquids, the pattern has a more oscillatory structure that contains spatial information in the form of the pair correlation function, $g(r)$. The spatial correlations are related to the observed structure factor, $S(Q)$, by the Fourier transform relation

$$
d(r)=4 \pi r \rho_{0}[g(r)-1]=\frac{2}{\pi} \int_{0}^{\infty} Q[S(Q)-1] \sin Q r \mathrm{~d} Q,
$$

where $Q$ is the scattering vector and $\rho_{0}$ is the number density. The real-space resolution is dependent on the maximum $Q$-value in the range of observation and is normally limited to about $20 \AA^{-1}$, giving a resolution of about $0.3 \AA$ (FWHM).

For disordered materials, the resolution is often quite important in distinguishing between different models of the structure but for crystalline materials there is less interest in obtaining a wide $Q$-range and more concern with the definition of the intensity and profiles of the peaks in $Q$-space. The nanotube structure represents an intermediate situation where the periodicity in the atomic correlations is affected by the curvature of the graphene sheets. The diffraction pattern therefore has a number of complex features, as discussed in Sec. 4.1.

Another aspect of the diffraction pattern for partially-ordered materials concerns the width of the Bragg peaks arising from the finite size of the crystal. If the crystallites are small, the peak is broadened in inverse proportion to the mean size of the crystals and can be used to estimate the effective coherence length within the periodic structure. In the case of activated carbons, this broadening effect may be very large. 


\subsection{Experimental facilities}

The requirements for the study of carbon nanotubes and related materials are more related to those for the study of disordered materials than for crystalline materials. Although it is sometimes necessary to investigate the shape of the diffraction peaks in some detail, it is more important to have a wide $Q$-range to improve the spatial resolution of the $d(r)$ function extracted from the observations. This feature is achieved by using short wavelength incident radiation.

In the case of neutrons, an incident wavelength of $0.7 \AA$ is adequate for most purposes and can be readily obtained from the hot source of a research reactor such as that at the Institut Laue Langevin [6]. However, the $Q$-range can be extended from $16 \AA^{-1}$ to $50 \AA^{-1}$ using the pulsed neutron technique, based on a spallation neutron source such as ISIS [7], at the Rutherford Appleton Lab. A typical plot for an activated carbon is shown in Fig. 1, which indicates that there is information in the diffraction pattern up to $40 \AA^{-1}$. An analysis of the resulting $d(r)$ data [8] shows that the first $\mathrm{C}-\mathrm{C}$ peak is split and the local carbon bond length within the layer is not singular. This important finding has important implications for the structural features of the graphene sheet and is discussed in another paper presented at this meeting [9]. A corresponding measurement for carbon nanotubes is planned but has not yet been conducted. One of the disadvantages of neutron measurements is that a relatively large quantity of material is needed in order to obtain the required statistical precision, especially at high $Q$-values. However, carbon has a small absorption cross-section and very little incoherent scattering, so the situation is quite favourable from other considerations.

$\mathrm{X}$-ray diffraction is an alternative method to use but there are limitations due to the atomic form-factor, which reduces the coherent contribution at large $Q$-values. This effect is coupled to the problem of subtracting the Compton scattering distribution. The availability of synchrotron radiation now offers two major advantages over laboratory-based equipment. The first is the very high intensity of the incident beam and the ability to use very small quantities of sample material and the second is the possibility of using short wavelengths. The technique of high-energy X-ray diffraction is currently under development and is of particular relevance to the investigation of carbon nanotubes. The instrument ID15 [10] at the European Synchrotron Radiation Facility, ESRF, is expected to play a major role in this area of development.

\section{Some experimental results}

The following sub-sections illustrate some of the recent diffraction measurements:

\subsection{Neutron diffraction from different forms of carbon}

The diffraction pattern from carbon nanotubes has some characteristics that relate to graphite and some that relate to activated carbons, as illustrated in Fig. 1. The (002) and (004) reflections for graphite correspond to the inter-layer spacing and are present for the nanotubes but displaced to lower $Q$-values, indicating an 


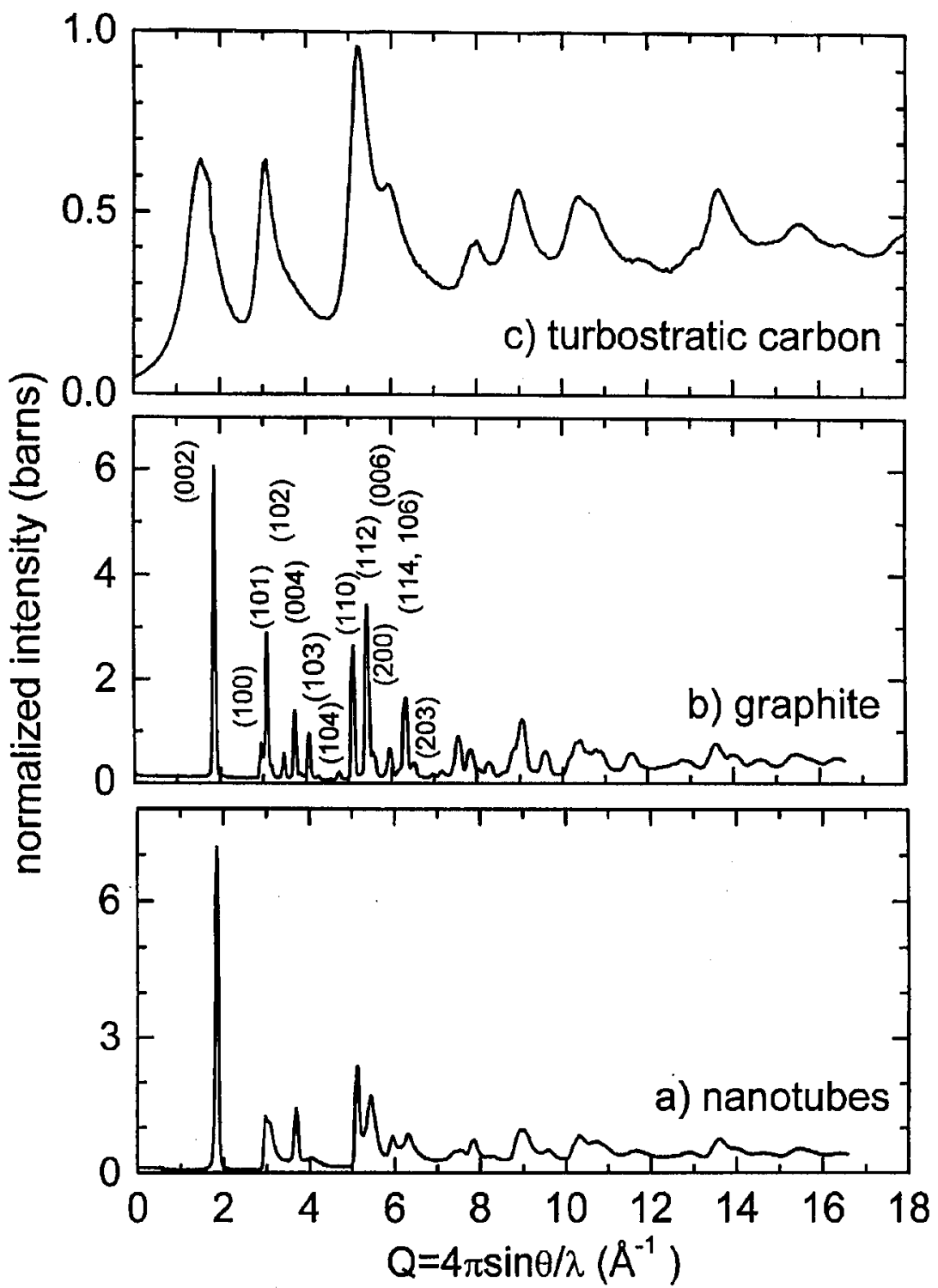

Fig. 1. Neutron diffraction measurements of the structure factor for (a) carbon nanotube, (b) graphite, and (c) activated carbon samples.

increase in the spacing. The $(h k 0)$ peaks for the nanotubes all have an asymmetric profile as expected [11] and there are no $(h k l)$ peaks due to the lack of correlations between layers, resembling the relative disorder of turbostratic graphite. In these measurements there is some residual intensity for the $(h k l)$ peaks, which is believed to come from some nanoparticles in the sample. 


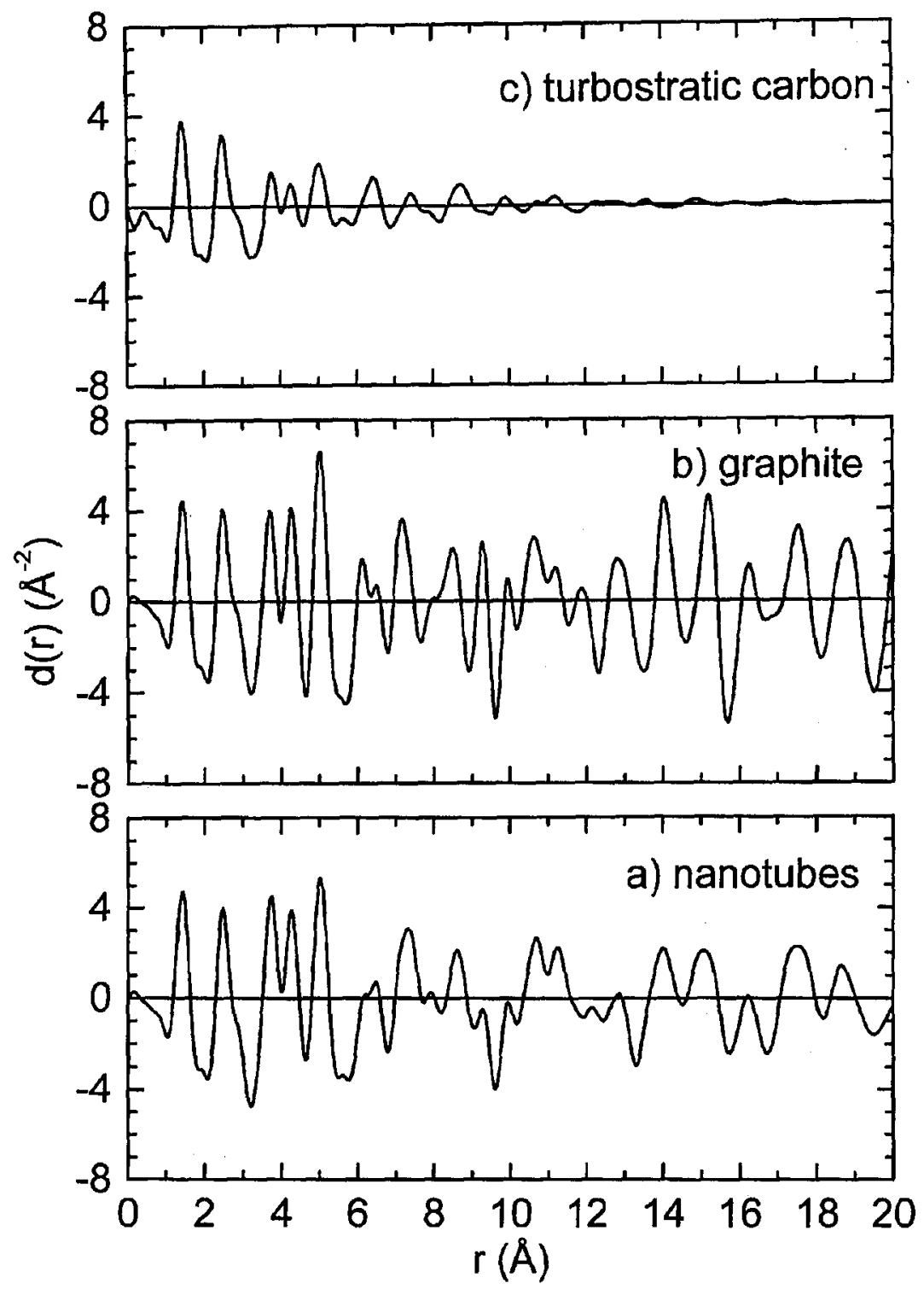

Fig. 2. The spatial correlation function, $d(r)$, evaluated from the data in Fig. 1.

The corresponding $d(r)$ functions are shown for these measurements in Fig. 2. It is notable that all the samples show local structure (1-4 $\AA$ ) which is almost identical, indicating the presence of the basic hexagonal configuration. At distances $>6 \AA$, the nanotube pattern departs from that of graphite, presumably due to the effects of curvature on the graphene sheets but it returns at longer distances due to correlations along the tube axis. In contrast, the activated carbon has little long-range order. A detailed analysis of the nanotube structure is in progress 
and should form a good basis for comparison of samples prepared by different techniques which are already known to give different diffraction patterns.

\subsection{X-ray measurements with synchrotron radiation}

The use of high-energy diffraction techniques with synchrotron radiation is illustrated in Fig. 3. The measured intensity is shown with the calculated atomic form-factor and the contribution from the Compton scattering. It is seen that the measurements can be made up to a $Q$-value of about $25 \AA^{-1}$ but care is needed in the conversion to a structure factor, $S(Q)$. A typical scan with a single detector enables the diffraction pattern to be obtained in about $2 \mathrm{~h}$ but a new system is under development using an area detector and recent tests [12] suggest that an equivalent pattern could be measured in only three minutes! This remarkable development has the capability of revolutionising the study of these materials but it will remain necessary to investigate all the experimental and analytical corrections to obtain accurate and reliable structural information. The complementarity of the neutron and X-ray methods will be important in establishing these new techniques.

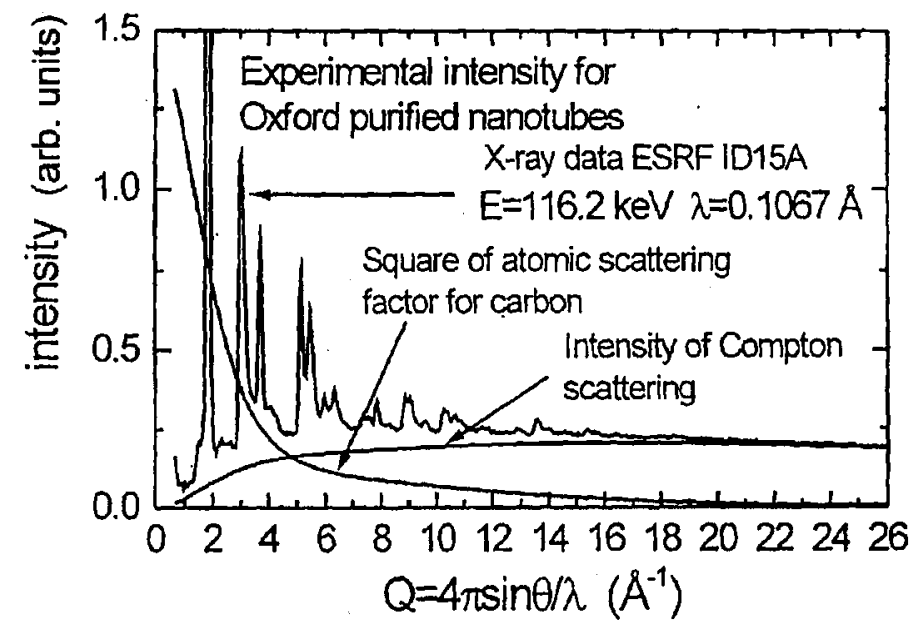

Fig. 3. High energy diffraction studies of carbon nanotubes showing the effects of the Compton scattering and the atomic form-factor.

\subsection{X-ray studies of carbon transformation involving carbon onions}

Synchrotron X-rays have been used in the study of four samples of heat-treated diamond nanoparticles obtained from Japan [5]. The diffraction patterns are shown in Fig. 4 as a function of scattering angle and the sample information is given in the caption. The pattern for sample $A$ exhibits several peaks corresponding to the diamond structure and the large widths are the result of particle size distribution and possible variation in the mean lattice spacing arising from stress. After heat treatment, $(B)$, the outer region of the particles is converted to a graphitic form and there is a consequent reduction in the intensity of these 


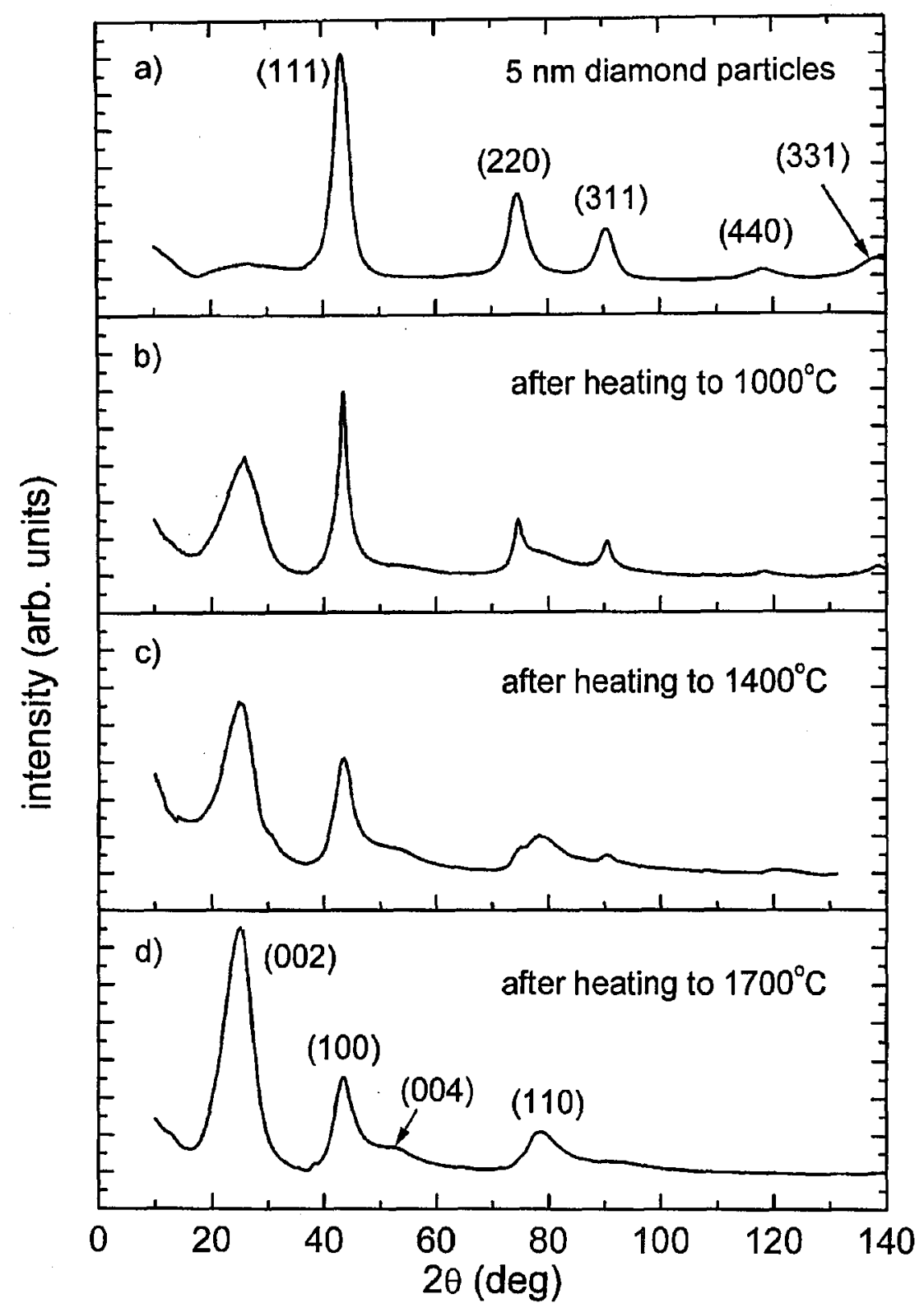

Fig. 4. X-ray diffraction studies of the transformation of diamond nanoparticles to carbon onions by heat treatment. Sample: (a) $5 \mathrm{~nm}$ diamond particles, (b) after heating to $1000^{\circ} \mathrm{C}$, (c) after heating to $1400^{\circ} \mathrm{C}$, (d) after heating to $1700^{\circ} \mathrm{C}$.

peaks, which also develop a sharper profile, suggesting that the smaller particles are converted more readily than the larger ones. There is also an additional peak at about $26^{\circ}$ that is characteristic of the (002) peak for graphene layers but it is 
much broader than for usual nanotube samples. The third sample $(C)$ corresponds to the formation of nano-onions. There is a further growth of the (002) peak, which remains quite broad, and there is evidence of residual diamond structure shown by the small peaks in the (220) and (311) positions. The position of the (111) diamond peak corresponds to that of the (100) peak for graphite so the observed intensity is due to both components. It is also notable that the characteristic asymmetry has developed through the emergence of a shoulder on the high-angle side of this peak and a new broad peak has emerged at $78^{\circ}$ that corresponds to the (110) peak of the graphene sheet. After further heat treatment, $(D)$, the graphite characteristics become more developed and the last vestiges of diamond disappear from the diffraction pattern.

These results show the transformation of the $s p^{3}$ carbon to $s p^{2}$ carbon through the temperature sequence and are in excellent agreement with the changes seen in the corresponding HRTEM images shown in Ref. [5]. A further analysis of the diffraction pattern is in progress and complementary measurements using neutrons have already been made. The modelling of the nano-onion structure poses several tantalising questions as the graphene sheets would seem to require curvature in two different planes, unlike the situation for the nanotubes. Additional measurements covering a wide $Q$-range are also planned.

\section{Conclusions and further developments}

This short review of techniques for studying the structure of various new carbon materials has shown how the use of modern diffraction methods can open up new possibilities for the detailed characterisation of spatial correlations in atomic systems. The unusual nature of the nanotubes and nano-onions will require both analytic and modelling developments to fully exploit the new opportunities and to understand the stable configurations. One possibility, currently under investigation, concerns the introduction of defects into the graphene sheet by the rotation of a single $\mathrm{C}-\mathrm{C}$ bond, as shown in Fig. 5. This change creates two 5- and 7-membered rings and could lead to a local distortion of the planarity. If present insignificant
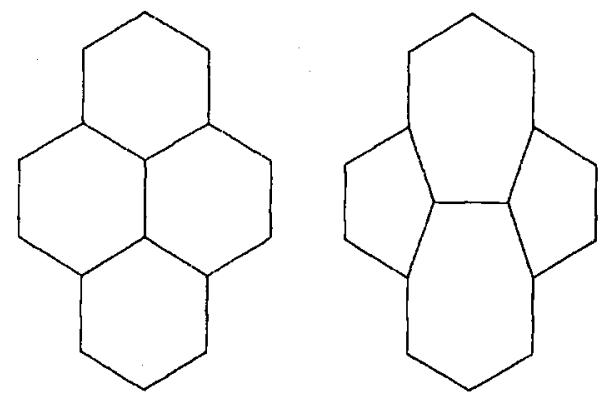

Fig. 5. A bonding defect in a hexagonal lattice showing the formation of 5- and 7-membered rings. 
amounts, the graphene sheet would adopt a wavy shape which could explain the microporosity features of activated carbons.

It is also already apparent that additional studies can be conducted on other materials that can now be grown inside the tubes as confined and encapsulated solids. The methods developed in the present studies can and will be readily extended to the investigation of structural modification for nanowires and other geometrically-restricted solids relative to that of the bulk material. This research field seems to be one that is at present in its infancy and is likely to develop rapidly through the effective use of international facilities. It is pertinent to add that Poland is expected to become a member of the ESRF group in the near future and will be able to play a role in the future use of these facilities.

\section{Acknowledgments}

We wish to acknowledge support for access to the various international facilities used in this research programme (ILL, ESRF) and to Henry Fischer, Veijo Honkimaki and David LeBolloc'k for assistance during the experiments. One of us (J.C.D.) would like to thank the organisers for an invitation to participate in the conference at Jaszowiec.

\section{References}

[1] A. Burian, J.C. Dore, H.E. Fischer, J. Sloan, Phys. Rev. B 59, 1665 (1999).

[2] S. Lazarescu, Ph. Lambin, P.A. Thiry, A. Fonseca, J.B. Nagy, A.A. Lucas, Phys. Rev. B 56, 12490 (1997).

[3] T. Kyotani, Li-fu Tsai, A. Tomita, Chem. Mater. 8, 2109 (1996).

[4] Y. Ando, X. Zhao, M. Ohkohchi, Jpn. J. Appl. Phys. 37, L61 (1998).

[5] S. Tomita, M. Hikita, M. Fujii, S. Hayashi, K. Yamamoto, Chem. Phys. Lett. 316, $361(2000)$.

[6] http://www.ill.fr.

[7] http://www.ISIS.uk .

[8] A. Burian, A. Ratuszna, J.C. Dore, W.S. Howells, Carbon 36, 1613 (1998).

[9] A. Burian, J.C. Dore, Acta Phys. Pol. A 98, 457 (2000).

[10] http://www.esrf.fr.

[11] S. Amelinckx, A.A. Lucas, Ph. Lambin, Rep. Prog. Phys. 62, 1471 (1999).

[12] V. Honkimaki, private communication. 